\section{Discrimination of Clinacanthus nutans extracts and correlation with antiplasmodial activity using ATR-FTIR fingerprinting}

By: Hashim, NHN (Hashim, Noor Haslinda Noor $)^{[1,2]}$; Ali, AH (Ali, Amatul Hamizah) ${ }^{[3]}$; Khatib, A (Khatib, Alfi) $)^{[4]}$; Latip, J (Latip, Jalifah) ${ }^{[1]}$

\section{VIBRATIONAL SPECTROSCOPY}

Volume: 104

Article Number: 102966

DOI: $10.1016 /$ j.vibspec.2019.102966

Published: SEP 2019

Document Type: Article

View Journal Impact

\section{Abstract}

Clinacanthus nutans or Sabah Snake Grass is a small shrub indigenous to tropical Asia. It has been used in the treatment of skin rashes, insect and snake bites, cancer and diabetes. In this study, Fourier transform infrared spectroscopy was used to discriminate the effects of different extraction methods on the chemical composition of $C$. nutans freeze dried leaves and stems. The samples were extracted using different methods (soaking and sonication) followed by the measurement of its antiplasmodial activity. Based on the absorption peaks, C. nutans extracts comprise of compounds with hydroxyl, methyl, sulfoxide, sulfone, aromatic and carbonyl functionalities which indicate the presence of carbohydrates, terpenes, sulfurous glycosides and aromatic compounds in the extracts. Multivariate data analysis such as orthogonal partial least square (OPLS) was used to correlate the FTIR spectra of the extracts with antiplasmodial activity. The OPLS model exhibited (RY)-Y-2 and Q(2)Y values of 0.896 and 0.736 , respectively. The RMSEE and RMSECV value were 22.41 and 32.36. The loading line plot of the OPLS model revealed that $\mathrm{OH}, \mathrm{C}=\mathrm{O}, \mathrm{C}-\mathrm{H}$ and $\mathrm{C}-\mathrm{C}$ functional group induced the activity, whereas, $\mathrm{C}-\mathrm{N}$ and $\mathrm{S}=\mathrm{O}$ reduced the bioactivity. These preliminary results demonstrated that FTIR spectroscopy can be used as a simple and rapid technique to discriminate C. nutans extracts obtained from different extraction methods which is useful in the quality control during processing of this plant.

\section{Keywords}

Author Keywords: Clinacanthus nutans; Fourier transform infrared spectroscopy (FTIR); Antiplasmodial; Metabolomics; Multivariate data analysis KeyWords Plus: TRANSFORM INFRARED-SPECTROSCOPY; ULTRASONICALLY ASSISTED EXTRACTION; BIOACTIVE PRINCIPLES; BARLERIA-LUPULINA; CULTIVARS; CONSTITUENTS; LEAVES

\section{Author Information}

Reprint Address: Latip, J (reprint author)

+ Univ Kebangsaan Malaysia, Fac Sci \& Technol, Ctr Adv Mat \& Renewable Resources, Bangi, Malaysia. Addresses:

+ [1] Univ Kebangsaan Malaysia, Fac Sci \& Technol, Ctr Adv Mat \& Renewable Resources, Bangi, Malaysia

+ [2] Int Islamic Univ Malaysia, Dept Plant Sci, Kulliyyah Sci, Kuantan, Malaysia

+ [3] Univ Kebangsaan Malaysia, Fac Sci \& Technol, Ctr Biotechnol \& Funct Food, Bangi, Malaysia

+ [4] Int Islamic Univ Malaysia, Dept Pharmaceut Chem, Kulliyyah Pharm, Kuantan, Malaysia

E-mail Addresses: jalifah@ukm.edu.my

\section{Funding}

\begin{tabular}{|l|l|}
\hline Funding Agency & Grant Number \\
\hline Universiti Kebangsaan Malaysia (UKM) & ETP 2013-063 \\
\hline
\end{tabular}

View funding text

Publisher

ELSEVIER, RADARWEG 29, 1043 NX AMSTERDAM, NETHERLANDS

Journal Information

Impact Factor: Journal Citation Reports

\section{Categories / Classification}

Research Areas: Chemistry; Spectroscopy

Web of Science Categories: Chemistry, Analytical; Chemistry, Physical; Spectroscopy

\section{Citation Network}

In Web of Science Core Collection

0

Times Cited

Create Citation Alert

\section{6}

Cited References

View Related Records

Use in Web of Science

Web of Science Usage Count

1 1

Last 180 Days $\quad$ Since 2013

Learn more

This record is from:

Web of Science Core Collection

- Science Citation Index Expanded

Suggest a correction

If you would like to improve the quality of the data in this record, please suggest a correction.

See more data fields 
1. Clinacanthus nutans: A review of the medicinal uses

Times Cited: 39

By: Alam, A.

pharmacology and phytochemistry Volume: 9 Pages: $402-409$ Published: 2016

2. Title: [not available]

Times Cited: 1

By: Bakar, S.Z.A.; Latip, J.; bin Din, L.; et al.

Metabolic Fingerprinting of Lichen Usnea baileyi by Fourier Transform Infrared Spectroscopy Volume: 452 Pages: $452-455$ Published: 2014

[Show additional data]

3. Recent advances in malaria drug discovery

Times Cited: 119

By: Biamonte, Marco A.; Wanner, Jutta; Le Roch, Karine G.

BIOORGANIC \& MEDICINAL CHEMISTRY LETTERS Volume: 23 Issue: 10 Pages: 2829-2843 Published: MAY 152013

4. Ultrasonically assisted Extraction of bioactive principles from Quillaja Saponaria Molina

Times Cited: 19

By: Cares, M. G.; Vargas, Y.; Gaete, L.; et al.

INTERNATIONAL CONGRESS ON ULTRASONICS, PROCEEDINGS Book Series: Physics Procedia Volume: 3 Issue: 1 Pages: 169-178 Published: 2010

5. Metabolomics: What You See is What You Extract

Times Cited: 22

By: Choi, Young Hae; Verpoorte, Robert

PHYTOCHEMICAL ANALYSIS Volume: 25 Issue: 4 Special Issue: SI Pages: 289-290 Published: JUL-AUG 2014

6. CONSTITUENTS OF CLINACANTHUS-NUTANS AND CRYSTAL-STRUCTURE OF LUP-20(29)-ENE-3-ONE

Times Cited: 33

By: DAMPAWAN, P; HUNTRAKUL, C; REUTRAKUL, V; et al.

JOURNAL OF THE SCIENCE SOCIETY OF THAILAND Volume: 3 Issue: 1 Pages: 14-26 Published: 1977

7. Rapid investigation of alpha-glucosidase inhibitory activity of Phaleria macrocarpa extracts using FTIR-ATR based fingerprinting

Times Cited: 12

By: Easmin, Sabina; Sarker, Md. Zaidul Islam; Ghafoor, Kashif; et al.

JOURNAL OF FOOD AND DRUG ANALYSIS Volume: 25 Issue: 2 Pages: 306-315 Published: APR 2017

8. Title: [not available]

Times Cited: 366

By: ERIKSSON L

MULTI MEGAVARIATE 1 Published: 2006

9. Kinetics extraction modelling and antiproliferative activity of clinacanthus nutans water extract

Times Cited: 1

By: Fazil, F. Nadiah Mohd; Azzimi, N. Syarafina Mohd; Yahaya, B. Hisham; et al.

The Scientific World Journal Volume: 2016 Published: 2016

[Show additional data]

10. Discrimination and characterisation of extra virgin olive oils from three cultivars in different maturation stages using Fourier transform infrared spectroscopy in tandem with chemometrics

By: Gouvinhas, Irene; de Almeida, Jose M. M. M.; Carvalho, Teresa; et al.

FOOD CHEMISTRY Volume: 174 Pages: 226-232 Published: MAY 12015

11. Direct discrimination of different plant populations and study on temperature effects by Fourier transform infrared spectroscopy

By: Khairudin, Khairunisa; Sukiran, Nur Afiqah; Goh, Hoe-Han; et al.

METABOLOMICS Volume: 10 Issue: 2 Pages: 203-211 Published:APR 2014

12. Phytochemical diversity of Clinacanthus nutans extracts and their bioactivity correlations elucidated by NMR based metabolomics

By: Khoo, Leng Wei; Mediani, Ahmed; Zolkeflee, Nur Khaleeda Zulaikha; et al.

PHYTOCHEMISTRY LETTERS Volume: 14 Pages: 123-133 Published: DEC 2015

13. Rapid discrimination of commercial strawberry cultivars using Fourier transform infrared spectroscopy data combined by multivariate analysis By: Kim, Suk Weon; Min, Sung Ran; Kim, Jonghyun; et al.

PLANT BIOTECHNOLOGY REPORTS Volume: 3 Issue: 1 Pages: 87-93 Published: FEB 2009

14. Nutritional Values and Amino Acid Profiles of Clinacanthus nutans (Belalai Gajah/Sabah Snake Grass) from Two Farms in Negeri Sembilan, Malaysia By: Kong, H. S.; Sani, Abdullah N.

Pertanika Journal of Tropical Agricultural Science Volume: 40 Issue: 4 Pages: 639-652 Published: NOV 2017

15. Clinacanthus nutans (Belalai Gajah / Sabah Snake Grass): antioxidant optimization on leaves and stems By: Kong, H.S.; Musa, K.H.; Sani, N. Abdullah

AIP C P Pages: 1784 Published: 2016

16. Efficacy of Clinacanthus nutans extracts in patients with herpes infection: Systematic review and meta-analysis of randomised clinical trials

By: Kongkaew, Chuenjid; Chaiyakunapruk, Nathorn

COMPLEMENTARY THERAPIES IN MEDICINE Volume: 19 Issue: 1 Pages: 47-53 Published: FEB 2011 
18. Discrimination of cultivation ages and cultivars of ginseng leaves using Fourier transform infrared spectroscopy combined with multivariate analysis By: Kwon, Yong-Kook; Ahn, Myung Suk; Park, Jong Suk; et al.

JOURNAL OF GINSENG RESEARCH Volume: 38 Issue: 1 Pages: 52-58 Published: JAN 2014

19. Discrimination of different genuine Danshen and their extracts by Fourier transform infrared spectroscopy combined with two-dimensional correlation infrared spectroscopy

By: Liu, Xin-hu; Xu, Chang-hua; Sun, Su-qin; et al.

SPECTROCHIMICAACTA PART A-MOLECULAR AND BIOMOLECULAR SPECTROSCOPY Volume: 97 Pages: 290-296 Published: NOV 2012

20. PARASITE LACTATE-DEHYDROGENASE AS AN ASSAY FOR PLASMODIUM-FALCIPARUM DRUG-SENSITIVITY

BY: MAKLER, MT; RIES, JM; WILLIAMS, JA; et al.

AMERICAN JOURNAL OF TROPICAL MEDICINE AND HYGIENE Volume: 48 Issue: 6 Pages: 739-741 Published: JUN 1993

21. Chemometric discrimination of genetically modified Coffea arabica cultivars using spectroscopic and chromatographic fingerprints

By: Moreira, Ivanira; Scarminio, leda Spacino

TALANTA Volume: 107 Pages: 416-422 Published: MAR 302013

22. Title: [not available]

By: Pavia, D.L.; Lampman, G.M.; Kriz, G.S.; et al.

Introduction to spectroscopy Published: 2009

Publisher: Brooks/Cole Cengage learning, USA

[Show additional data]

23. Bioactive constituents from the leaves of Clinacanthus nutans Lindau

Times Cited: 70

By: Sakdarat, Santi; Shuyprom, Aussavashai; Pientong, Chamsai; et al.

BIOORGANIC \& MEDICINAL CHEMISTRY Volume: 17 Issue: 5 Pages: 1857-1860 Published: MAR 12009

24. Title: [not available]

By: SHIM SY

INT J INTEGR BIOL Volume: 14 Pages: 7 Published: 2013

25. Use of FT-IR Spectra and PCA to the Bulk Characterization of Cell Wall Residues of Fruits and Vegetables Along a Fraction Process

By: Szymanska-Chargot, Monika; Zdunek, Artur

FOOD BIOPHYSICS Volume: 8 Issue: 1 Pages: 29-42 Published: MAR 2013

26. Sulfur-containing glucosides from Clinacanthus nutans

By: Teshima, KI; Kaneko, T; Ohtani, K; et al.

PHYTOCHEMISTRY Volume: 48 Issue: 5 Pages: 831-835 Published: JUL 1998

27. C-Glycosyl flavones from Clinacanthus nutans

Times Cited: 26

By: Teshina, Ko-Ichiro; Kaneko, Tetsuo; Ohtani, Kazuhiro; et al.

Natural Medicines Volume: 51 Issue: 6 Pages: 557 Published:Dec., 1997

28. The Application of SIMCA P+ in Shotgun Metabolomics Analysis of ZIC\&REG; HILIC-MS Spectra of Human urine-Experience with the Shimadzu IT-T of and profiling Solutions Data extraction Software

By: Trivedi, D. K.; lles, R. K.

J. Chromatogr. Sep. Tech. Volume: 3 Pages: 1-5 Published: 2012

CrossRef

29. Chemical Constituents and Bioactivities of Clinacanthus nutans Aerial Parts

Times Cited: 28

By: Tu, Shu-Fen; Liu, Rosa Huang; Cheng, Yuan-Bin; et al.

MOLECULES Volume: 19 Issue: 12 Pages: 20382-20390 Published: DEC 2014

30. Sulfur-containing compounds from Clinacanthus siamensis

Times Cited: 10

By: Tuntiwachwuttikul, P; Pootaeng-on, Y; Pansa, P; et al.

CHEMICAL \& PHARMACEUTICAL BULLETIN Volume: 51 Issue: 12 Pages: 1423-1425 Published: DEC 2003 